\title{
Correction to: Multi-institutional analysis of CT and MRI reports evaluating indeterminate renal masses: comparison to a national survey investigating desired report elements
}

Eric M. Hu, ${ }^{1,2}$ Andrew Zhang, ${ }^{1,2}$ Stuart G. Silverman, ${ }^{3,9}$ Ivan Pedrosa, ${ }^{4,9}$

Zhen J. Wang, ${ }^{5,9}$ Andrew D. Smith,,${ }^{6,9}$ Hersh Chandarana, ${ }^{7,9}$ Ankur Doshi, ${ }^{7,9}$

Atul B. Shinagare, ${ }^{3,9}$ Erick M. Remer, ${ }^{8,9}$ Samuel D. Kaffenberger, ${ }^{1,10}$ David C. Miller, ${ }^{1,10}$ Matthew S. Davenport ${ }^{1,2,9,10,11}$

${ }^{1}$ Michigan Medicine, Ann Arbor, MI, USA

${ }^{2}$ Michigan Radiology Quality Collaborative, Ann Arbor, MI, USA

${ }^{3}$ Brigham and Women's, Boston, MA, USA

${ }^{4}$ UT Southwestern, Dallas, TX, USA

${ }^{5}$ UCSF, San Francisco, CA, USA

${ }^{6} \mathrm{UAB}$, Birmingham, AL, USA

${ }^{7}$ NYU, New York, NY, USA

${ }^{8}$ Cleveland Clinic, Cleveland, OH, USA

${ }^{9}$ Society of Abdominal Radiology Disease-Focused Panel on Renal Cell Carcinoma, Ann Arbor, MI, USA

${ }^{10}$ Michigan Urological Surgery Improvement Collaborative, Ann Arbor, MI, USA

${ }^{11}$ Department of Radiology, 1500 E. Medical Center Dr. B2-A209P, Ann Arbor, MI 48108, USA

Correction to: Abdom Radiol (2018) https://doi.org/10.1007/s00261-018-1609-x

In the original version of this article one author name was published incorrectly. The co-author's name was published as Ivan M. Pedrosa, instead it should be Ivan Pedrosa. The original article has been corrected.

The original article can be found online at https://doi.org/10.1007/s00 261-018-1609-x.

Correspondence to: Matthew S. Davenport; email: daven@med.umich.edu 